\title{
Efficacy and safety of low-dose tacrolimus for active rheumatoid arthritis with an inadequate response to methotrexate
}

\author{
Won-Seok Lee ${ }^{1}$, Sang-Il Lee ${ }^{2}$, Myeung-Soo Lee ${ }^{3}$, Sung-Il Kim ${ }^{4}$, Shin-Seok Lee ${ }^{5}$, and Wan-Hee Yoo ${ }^{1}$
}

${ }^{1}$ Division of Rheumatology, Department of Internal Medicine, Research Institute of Clinical Medicine of Chonbuk National University-Biomedical Research Institute of Chonbuk National University Hospital, Jeonju; ' ${ }^{2}$ Division of Rheumatology, Department of Internal Medicine, Gyeongsang National University Hospital, Jinju; ${ }^{3}$ Division of Rheumatology, Department of Internal Medicine, Wonkwang University Hospital, Iksan; ' ${ }^{2}$ ivision of Rheumatology, Department of Internal Medicine, Pusan National University Hospital, Busan; ${ }^{5}$ Division of Rheumatology, Department of Internal Medicine, Chonnam National University Hospital, Gwangju, Korea

Received: March 10, 2015

Revised : June 8, 2015

Accepted: June 19, 2015

\section{Correspondence to}

Wan-Hee Yoo, M.D.

Division of Rheumatology,

Department of Internal Medicine,

Chonbuk National University

Medical School and Research

Institute of Clinical Medicine

of Chonbuk National University

Hospital, 20 Geonji-ro, Deok-

jin-gu, Jeonju 54907, Korea

Tel: +82-63-250-1672

Fax: +82-63-254-1609

E-mail:ywhim@jbnu.ac.kr

Background/Aims: To determine the efficacy and safety of low-dose tacrolimus in Korean rheumatoid arthritis (RA) subjects with an inadequate response to methotrexate (MTX).

Methods: This was a multicenter, open-label study conducted at five Korean sites. Fifty-six patients with active RA, despite treatment for $\geq 1$ month with a stable, maximally tolerated dosage of oral MTX (median dosage, $15 \mathrm{mg} / \mathrm{wk}$ ), were enrolled and received $1.5 \mathrm{mg} /$ day of tacrolimus as a single oral dose once per day for 16 weeks while continuing to receive MTX. All other disease-modifying anti-rheumatic drugs were discontinued, whereas stable dosages of nonsteroidal anti-inflammatory drugs and oral corticosteroids $(\leq 10 \mathrm{mg} /$ day of prednisone or an equivalent corticosteroid) were allowed. The primary clinical response criterion was the American College of Rheumatology's definition of $20 \%$ improvement (ACR2O) at the end of treatment.

Results: The ACR20 response rate was $42.9 \%$ (24 of 56 patients) in patients who had received tacrolimus at least once. The overall ACR50 and ACR70 responses at the end of treatment for all patients were $30.4 \%$ and $10.7 \%$, respectively. Throughout the treatment period, 37 patients experienced 71 adverse events (AEs) in total, and four patients left the study because of AEs. In addition, 15 patients in total experienced treatment-related AEs. Throughout the treatment period, two patients were reported to experience two serious AEs, and one patient left the study because of a serious AE.

Conclusions: In patients whose active RA persists despite treatment with MTX, low-dose tacrolimus in combination with MTX appears to be safe and well tolerated, and provides clinical benefit.

Keywords: Arthritis, rheumatoid; Tacrolimus; Methotrexate

\section{INTRODUCTION}

Rheumatoid arthritis (RA) is a chronic inflammatory disease of unknown etiology characterized by symmetric, peripheral polyarthritis. It is the most common form of chronic inflammatory arthritis, and often results in joint damage and physical disability. The cause of RA is unknown, and the prognosis is guarded. However, advances in understanding its pathogenesis have 
fostered the development of new therapeutics, resulting in improved outcomes. The current treatment strategy, which reflects this progress, is to initiate aggressive therapy immediately after diagnosis and to escalate this therapy, based on an assessment of disease activity, in pursuit of clinical remission.

The safety profile of methotrexate (MTX) has made earlier treatment possible, and most clinicians now prescribe MTX for RA patients. However, strong evidence regarding the appropriate treatment for patients who show an inadequate response to MTX is lacking [1-3].

Tacrolimus is a potent macrolide calcineurin inhibitor produced by the bacterium Streptomyces tsukubaensis. Through its inhibition of calcineurin activity, it is capable of down-regulating the synthesis of various inflammatory cytokines, including tumor necrosis factor- $\alpha$, interleukin-1 $\beta$ and -6 , and interferon-c, by activated T-cells by binding to the cytoplasmic FK binding protein [4]. T-cell involvement has been reported as a mechanism of RA pathogenesis [5]. Hence, considering that tacrolimus can inhibit the release of inflammatory cytokines involved in RA development by blocking T-cell activation, it has been developed as an anti-RA drug and was approved for sale in Europe and the United States in April 2005 after a series of clinical studies.

In Korea, this drug has recently been indicated as "treatment for some cases of RA where the existing drugs show no effect," and the efficacy of high-dose tacrolimus has been reported in various academic clinical studies [6-9]. However, there are currently few studies examining the effects of low-dose tacrolimus in these patients, and thus, based on these previous clinical studies, this study aimed to assess the efficacy of low-dose tacrolimus (1.5 mg) in Korean RA patients with an inadequate response to MTX.

\section{METHODS}

\section{Patients}

Fifty-six patients aged between 18 and 75 years who met the American College of Rheumatology (ACR) 1987 revised criteria for RA [10], whose disease persisted for $\geq$ 6 months, and who had active RA $(\geq 5$ tender/painful joints and $\geq 3$ swollen joints) at baseline were enrolled at five Korean centers for this 4-month, open-label study.
Patients who had been receiving oral MTX (12.5 to 20 $\mathrm{mg} / \mathrm{wk}$ ) at a stable dosage for $\geq 1$ month prior to study entry were considered eligible, and were continued on the same dosage of MTX throughout the study. All other disease-modifying anti-rheumatic drugs (DMARDs) were discontinued $\geq 8$ weeks prior to study entry. Patients were allowed to receive stable dosages of nonsteroidal anti-inflammatory drugs and oral corticosteroids $(\leq 10 \mathrm{mg} /$ day of prednisone or an equivalent corticosteroid) from 4 weeks prior to study entry. Intra- and/or periarticular steroid injections were allowed during the study period, but the injected joint was excluded from subsequent efficacy assessments. Exclusion criteria were pregnancy or lactation, liver disease (defined as levels of aspartate aminotransferase, alanine aminotransferase, alkaline phosphatase, or total bilirubin $>2$ times the upper limit of normal), serum creatinine (Cr) levels outside the normal range for the patient's age and sex, hemoglobin concentration $<9.0 \mathrm{mg} / \mathrm{dL}$, white blood cell count $<3,000 / \mathrm{mm}^{3}$, and platelet count $<100,000 / \mathrm{mm}^{3}$. The Institutional Review Board at each study site approved the protocol, and all patients provided written informed consent prior to any study-related procedures, which were performed in accordance with the principles of the Declaration of Helsinki.

\section{Treatment}

Patients were treated with $1.5 \mathrm{mg}$ of tacrolimus (1- and 0.5-mg capsules), administered orally once daily, in addition to each patient's current prescribed oral MTX dosage (highest tolerated MTX dosage $\leq 20 \mathrm{mg} / \mathrm{wk}$ ). The MTX dosage was lowered only if the patient experienced an adverse event (AE) that may have been related to MTX treatment. All patients received stable folate supplementation (1 mg/day).

\section{Assessment}

All patients who received at least one dose of the study drug were included in the safety and efficacy analyses. Clinical and laboratory assessments were performed at baseline, week 2, and months 1 to 4 . Efficacy was assessed at $0,4,8$, and 16 weeks using the ACR definitions of improvement; ACR20, ACR50, and ACR7o responses were defined as $\geq 20 \%, \geq 50 \%$, and $\geq 70 \%$ improvement, respectively, in the tender or painful joint and swollen joint counts [11]. 
The primary efficacy endpoint was the ACR2o response at the end of treatment. Secondary endpoints were the ACR5O and ACR70 responses, the acute-phase reactant (erythrocyte sedimentation rate [ESR] by the Westergren method or C-reactive protein [CRP] level), disease activity score (DAS) 28-ESR score, patient assessment of pain on a 100-mm visual analog scale (VAS), and patient assessment of physical function (based on the modified Korean Health Assessment Questionnaire [KHAQ]) at o, 4, 8, and 16 weeks after initiating the tacrolimus combination therapy.

Safety was evaluated by physical examinations, monitoring of vital signs and treatment-emergent AEs, and routine clinical laboratory testing (with emphasis on parameters that had previously shown elevations among transplantation patients who were treated with tacrolimus, i.e., serum Cr, blood urea nitrogen, glucose, and hemoglobin Alc). Patients with ongoing treatment-emergent AEs at the end of treatment were monitored until the event resolved or stabilized. Serum Cr levels were measured at $0,4,8$, and 16 weeks and/or at the end of treatment. Patients who had an increase in the serum $\mathrm{Cr}$ level of $\geq 40 \%$ from baseline or $\geq 30 \%$ from the previous visit, which was considered clinically significant, had the test repeated 1 week later. If the Cr level remained elevated, treatment with tacrolimus was withheld. If the elevation persisted, the patient was withdrawn from the study. If, in the investigator's opinion, the serum $\mathrm{Cr}$ level decreased to a sufficient level after tacrolimus was withheld, then the treatment could be reinstated within 2 weeks, and the patient was allowed to remain in the study. The Cr levels were not routinely monitored after the end of the study. A serious adverse event (SAE) was defined as an $\mathrm{AE}$ that resulted in any of the following outcomes: death, life-threatening AE, persistent or significant disability or incapacity, in-patient abnormality or birth defect, or an important medical event.

\section{Statistical analyses}

Safety and efficacy analyses were performed on the full analysis set, consisting of all patients who received at least one dose of the study drug. The 95\% confidence intervals (CIs) were calculated for the ACR response rates at the end of treatment, assuming a normal approximation to the binomial parameter. For continuous variables such as DAS28-ESR, ESR, CRP, and pain
VAS, the mean value and standard deviation at each time point along with the $95 \%$ CIs were calculated. To evaluate the presence of a statistically significant difference from baseline at each time point, the paired $t$ test was performed. A p value less than 0.05 was considered to indicate statistical significance.

\section{RESULTS}

\section{Demographics, baseline characteristics, and dispo- sition}

Of the 56 patients enrolled in the study, 43 (76.7\%) completed 4 months of treatment. Patients' demographic and baseline characteristics are shown in Table 1. Mean age of enrolled patients was 50.68 years. Thirteen patients were male and 43 were female. Their mean duration of RA was 71.16 months (range, 6 to 359). The majority of patients were classified as having ACR classes 2 and 3 RA (23 patients [41.0\%] and 20 [35.7\%], respectively), whereas 8 patients $(14.2 \%)$ and 5 (8.9\%) were classified as having classes 1 and 4 RA, respectively. At baseline, mean numbers of tender and swollen joints were 11.73 and 8.91, respectively.

\section{Efficacy}

The ACR2O response rate was $42.9 \%$ (24 of 56 patients) in patients who had received tacrolimus at least once, whereas it was $51.2 \%$ (22 of 43) in those who completed the study as per protocol. The overall ACR50 and ACR70 responses at the end of treatment for all patients were 30.4\% and 10.7\%, respectively (Table 2).

Compared to baseline, the value of DAS28-ESR showed significant differences at 4,8 , and 16 weeks, with mean changes of $-0.86,-1.04$, and -1.42 , respectively (Fig. 1A). At baseline, the mean ( \pm standard deviation) ESR and CRP were $55.95 \pm 29.29$ and $2.27 \pm 2.92$, respectively. Changes in the ESR from baseline at 4, 8, and 16 weeks were $-8.04,-11.42$, and -18.11 , respectively, which exhibited a stable decreasing trend. Similarly, mean changes in the CRP levels also showed a decreasing pattern; mean changes from baseline at 4,8 , and 16 weeks were -0.67 , -0.55 , and -1.35 , respectively (Fig. $1 B$ ). In terms of the pain, the change in VAS from baseline showed significant differences at 4, 8, and 16 weeks, with mean changes of $-13.09,-18.34$, and -25.66 , respectively (Fig. $1 \mathrm{C}$ ). The 

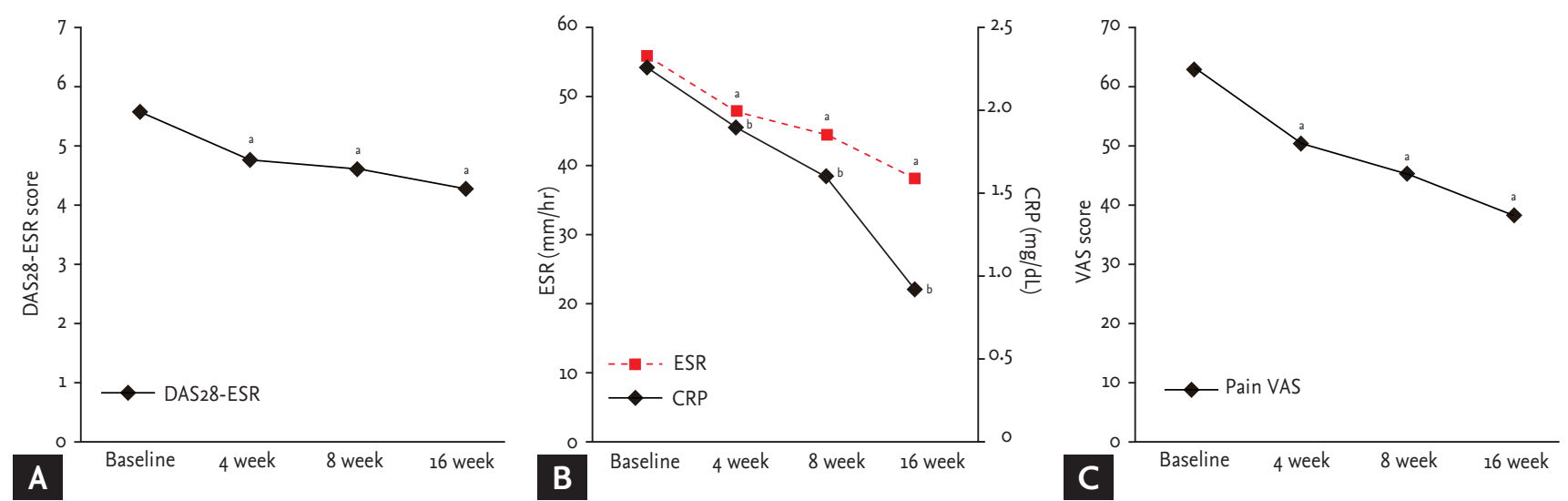

Figure 1. The Disease Activity Score for 28-joint counts (DAS28), erythrocyte sedimentation rate/C-reactive protein (ESR/CRP) and pain visual analog scale (VAS). Serial changes in the (A) DAS28-ESR score, (B) ESR/CRP, and (C) pain VAS score. The data are presented as the mean. ${ }^{a, b} p<0.05$, compared with baseline.

Table 1. Clinical baseline characteristics $(n=56)$

\begin{tabular}{lc}
\hline Characteristic & Value \\
\hline Age, yr & $50.68 \pm 11.96$ \\
Female sex & $43(76.7)$ \\
Time since diagnosis, mon & $71.16 \pm 79.58$ \\
Assessments & $11.73 \pm 9.81$ \\
\hline Tender joint count & $8.91 \pm 6.63$ \\
Swollen joint count & $63.03 \pm 25.74$ \\
\hline Patient pain on 10o-mm VAS & $5.59 \pm 1.05$ \\
DAS28 & $16.79 \pm 11.84$ \\
KHAQ & $55.95 \pm 29.29$ \\
\hline ESR, mm/hr & $2.27 \pm 2.92$ \\
\hline CRP, mg/dL & \\
\hline ACR functional classification, class & $8(14.2)$ \\
\hline 1 & $23(41.0)$ \\
2 & $20(35.7)$ \\
3 & $5(8.9)$ \\
\hline
\end{tabular}

Values are presented as the mean \pm SD or number (\%).

VAS, visual analog scale; DAS28, the Disease Activity Score for 28-joint counts; KHAQ, Korean version of Health Assessment Questionnaire; ESR, erythrocyte sedimentation rate; CRP, C-reactive protein; ACR, American College of Rheumatology.

mean KHAQ at baseline was 16.79, and the mean change from baseline at 16 weeks was -2.29 , which was statistically significant.
Table 2. American College of Rheumatology response ( $n=$ 56)

\begin{tabular}{lc}
\hline ACR response & No. $(\%)$ \\
\hline ACR20 & $24(42.9)$ \\
ACR50 & $17(30.4)$ \\
ACR70 & $6(10.7)$ \\
\hline
\end{tabular}

Percentage of patients achieving the American College of Rheumatology (ACR) definition of $20 \%, 50 \%$, and $70 \% \mathrm{im}$ provement in 16 weeks of treatment.

\section{Safety}

A summary of the AEs is provided in Table 3. Throughout the treatment period, 71 AEs in 37 patients were reported. The most frequent AEs, classified by organ system class, were gastrointestinal disorders (17 patients), infection and infestations (15), and general disorders and administration site conditions (10). The most frequent AEs, according to the preferred terms of the Medical Dictionary for Regulatory Activities version 12 (MedDRA), were upper respiratory tract infections (11 patients), diarrhea (5), epigastric discomfort (5), pain (4), face edema (3), and back pain (3).

In total, 15 patients experienced treatment-related AEs. The most frequent treatment-related AEs, classified by organ system class, were gastrointestinal disorders (9 patients), followed by general disorders and administration site conditions (6). When classified according to the preferred terms, diarrhea, epigastric discomfort, and face edema were the most frequent treatment-relat- 
Table 3. Summary of common treatment-emergent adverse events $\left(n=5^{6}\right)$

\begin{tabular}{|c|c|c|}
\hline System organ class preferred term & $\begin{array}{l}\text { Regardless of relationship } \\
\text { to tacrolimus }\end{array}$ & $\begin{array}{l}\text { Possibly or probably related } \\
\text { to tacrolimus }\end{array}$ \\
\hline No. with any adverse event & $37(66)$ & $15(27)$ \\
\hline Cardiac disorder & $1(2)$ & o \\
\hline Eye disorder & $3(5)$ & o \\
\hline Gastrointestinal disorder & $17(30)$ & $9(16)$ \\
\hline General disorder and administration site condition & $10(18)$ & $6(11)$ \\
\hline Infection and infestation & $15(27)$ & o \\
\hline Investigation & $1(2)$ & $1(2)$ \\
\hline Musculoskeletal and connective tissue disorder & $6(11)$ & o \\
\hline Nervous system disorder & $5(9)$ & $2(4)$ \\
\hline Respiratory, thoracic and mediastinal disorder & $2(4)$ & $1(2)$ \\
\hline Skin and subcutaneous tissue disorder & $1(2)$ & $\mathrm{O}$ \\
\hline
\end{tabular}

Values are presented as number (\%).

ed AEs, each reported by 3 patients.

Throughout the treatment period, two SAEs were reported in two patients; however, neither of these was treatment-related. The SAEs, as classified by system organ class, were infection and infestations, and musculoskeletal and connective tissue disorders. One of the patients experienced arthralgia and exacerbation of swelling, which resulted in hospitalization. This patient discontinued the study protocol because of the SAE. The other patient experienced acute bacterial enteritis, which also resulted in hospitalization. No subjects died because of an SAE.

During the study, four patients in total left the study because of AEs, including the aforementioned patient who left due to an SAE. Two of these patients experienced treatment-related AEs, whereas the remaining patient experienced a non-treatment-related AE. The first patient experienced arthralgia and exacerbation of swelling, which was reported as an SAE (MedDRA: musculoskeletal and connective tissue disorders). The patient discontinued the study protocol because of this SAE, which was not treatment-related. The second patient developed gingival hypertrophy (MedDRA: gastrointestinal disorders with gingival hypertrophy). This patient requested to be withdrawn from the study because of the AE. This AE was considered to be probably related to the treatment. The third patient reported pain and showed increased CRP and rheumatoid factor lev- els, both of which were indications for withdrawal from the study. Pain was considered to be possibly related to the treatment, and the increased CRP and rheumatoid factor levels were considered to be probably related to the treatment. The last patient experienced myalgia and myocardial ischemia and requested withdrawal from the study because of the $\mathrm{AE}$, which was determined not to be treatment-related.

\section{DISCUSSION}

It has been previously reported that use of single-agent tacrolimus or tacrolimus plus MTX is safe and effective in the treatment of RA. However, previous studies investigating combination therapy of tacrolimus plus MTX used a relatively high dose of tacrolimus ( $3 \mathrm{mg}$ ).

When efficacy in groups with $2 \mathrm{mg}$ of tacrolimus was compared with $3 \mathrm{mg}$ of tacrolimus, the 2-mg group showed an ACR20 response rate of 33\%, lower than that of the 3-mg group; however, for the ACR50 and ACR7o response rates, there was no difference between groups [8]. Furthermore, in a study with RA patients aged $\geq 65$, after checking improvement in RA symptoms by administering $1.5 \mathrm{mg}$ of tacrolimus and increasing the dose to $3 \mathrm{mg}$ only when necessary, $53.5 \%$ of patients showed improvements just with $1.5 \mathrm{mg}$ without increasing the dose to $3 \mathrm{mg}$ [12]. Thus, we planned this study under the 
notion that if a low dose (1.5 mg) can show sufficient efficacy, it would be more favorable in terms of safety.

This multicenter, open-label study assessed the safety and efficacy of low-dose tacrolimus in combination with oral MTX for the treatment of RA. Patients whose disease remained active despite treatment with their highest tolerated dosage of MTX were enrolled. After the study period, half of the patients showed clinical improvement because $1.5-\mathrm{mg} /$ day tacrolimus was added to their prescribed stable MTX dosage regimen, as measured by the ACR2o response rate. Overall, low-dose tacrolimus appeared generally safe and well tolerated by patients in this study.

Efficacy results for all patients in this open-label study demonstrated that $42.9 \%$ of patients achieved an ACR2O response rate, whereas $30.4 \%$ and $10.7 \%$ of patients achieved ACR50 and ACR70 response rates, respectively, with tacrolimus. The efficacy of tacrolimus use in RA has been previously studied in four double-blind randomized controlled trials (RCTs) (Table 4) [6-9,12-14]. The highest ACR2o response rate was seen in two Japanese studies $[6,7]$, in which it approached $50 \%$, whereas a lower rate of approximately $30 \%$ was found in two other studies conducted in the United States [8,9]. All of these RCTs, which incorporated different tacrolimus dose groups, demonstrated dose-dependent ACR2o response rates, with statistically significant differences in the ACR2o response rates observed among the 3-, 5-mg, and placebo groups in the study by Furst et al. [9]. However, interestingly, no statically significant differences were found between the placebo and low-dose groups $(1,1.5$, and $2 \mathrm{mg})$ in each study. Unlike these RCTs, the present study was an open-label, single-arm study. The ACR2o response rate of this study was $42.9 \%$, which is similar to those reported in the Japanese studies. However, the dosage used in this study was $1.5 \mathrm{mg}$ /day for all patients, which was relatively low when compared to both the previous RCTs and open label studies. With regard to the efficacy of this combination therapy, we found that the mean DAS28-ESR score also was improved. Furthermore, in contrast to cyclosporine, which has not been reported to result in improvements in the ESR $[15,16]$, low-dose tacrolimus treatment resulted in improvements in both the CRP level and ESR. Although we cannot be sure what caused the ACR2o response rate to be higher for the low dose, we can suppose that an
RA duration of 7 years, which is shorter than 11 years in the previous study, and racial difference, as it has never been studied in Korea, may influence this result. Also, it is considered that there are limitations to making a definite conclusion from the short duration of this study, thus a longer study with a larger group size should be conducted.

The incidence of AEs in this study was $66.1 \%$, and the rate of withdrawal due to AEs was $5.4 \%$. This rate was relatively low when compared to previous studies on the topic (Table 5) [6-9,12-14]. Gastrointestinal tract-related AEs were among the most commonly reported AEs across all tacrolimus dose groups in the four RCTs and in the open-label studies (Table 5), which is similar to the findings of our study. Among tacrolimus users, a higher incidence of AEs involving any system was reported in RCTs from the United States (80\% to $92.8 \%$ ) compared with RCTs from Japan (44.4\% to 65\%), and the open-label study by Yocum et al. [13] (59\%, possibly or probably related to tacrolimus). However, of note, a higher incidence of AEs was also seen in the placebo groups in RCTs in the United States, with an overall withdrawal rate due to AEs within the RCTs ranging between $4 \%$ and $16 \%$.

Our study has several limitations. First, compared to other open-label studies, our sample size was small and the treatment duration was short. However, we wish to determine the effectiveness of low-dose tacrolimus, and the results of this study will help in planning a largescale, long-term study. Second, no radiographic data were available, and hence, we could not assess structural damage. Nevertheless, our study duration may have been too short to identify radiographic changes, and so we did not assess changes in structural damage. Finally, the tacrolimus blood concentration was not measured. Further study is therefore needed to determine the blood concentration of tacrolimus with the administration of high-dose and low-dose tacrolimus.

According to the recommendations for treating RA patients proposed by the European League Against Rheumatism and other authorities [17,18], early initiation of DMARDs with the aim of remission or low disease activity while avoiding bone destruction is desirable. Thus, biologics are usually recommended for patients who have an inadequate response to MTX. However, many physicians hesitate to prescribe biologics to pa- 
Table 4. Summary of randomized, double-blind, controlled trials of tacrolimus in rheumatoid arthritis and this study

\begin{tabular}{|c|c|c|c|c|c|}
\hline Study & Country & $\begin{array}{l}\text { Duration, } \\
\text { wk }\end{array}$ & $\begin{array}{c}\text { No. of } \\
\text { patients }\end{array}$ & Dose group & $\begin{array}{c}\text { ACR2o response } \\
\text { rate, \% (mg) }\end{array}$ \\
\hline \multicolumn{6}{|c|}{ Randomized controlled study } \\
\hline Kawai et al. (2006) [6] & Japan & 28 & 204 & $\mathrm{TAC}_{3} \mathrm{mg} /$ day or mizoribine $150 \mathrm{mg}$ & $48.5(3)$ \\
\hline \multirow[t]{2}{*}{ Kondo et al. (2004) [7] } & Japan & 16 & 212 & TAC 1.5 or $3 \mathrm{mg} /$ day or placebo & $48.3(3)$ \\
\hline & & & & & $24.6(1.5)$ \\
\hline \multirow[t]{2}{*}{ Yocum et al. (2003) [8] } & USA & 24 & 464 & TAC 2 or $3 \mathrm{mg} /$ day or placebo & $32(3)$ \\
\hline & & & & & $21.4(2)$ \\
\hline \multirow[t]{3}{*}{ Furst et al. (2002) [9] } & USA & 24 & 268 & TAC 1,3 , or $5 \mathrm{mg} /$ day or placebo & $29(1)$ \\
\hline & & & & & $34 \cdot 4(3)$ \\
\hline & & & & & $50(5)$ \\
\hline \multicolumn{6}{|l|}{ Open-label study } \\
\hline Kawai et al. (2006) [12] & Japan & 28 & 57 & TAC 1.5 to $3 \mathrm{mg} /$ day & 46.3 (overall) \\
\hline Yocum et al. (2004) [13] & USA & 52 & 896 & TAC 2 or 3 mg/day & 38.4 (overall) \\
\hline Kremer et al. (2003) [14] & USA & 24 & 80 & $\mathrm{TAC}_{3} \mathrm{mg} /$ day & $52.5(3)$ \\
\hline Present study & Korea & 16 & 56 & TAC $1.5 \mathrm{mg} /$ day & $42.9(1.5)$ \\
\hline
\end{tabular}

ACR, American College of Rheumatology; TAC, tacrolimus.

Table 5. Summary of tacrolimus-related adverse events in rheumatoid arthritis studies and this study

\begin{tabular}{|c|c|c|c|c|}
\hline Study & $\begin{array}{l}\text { TAC dose, } \\
\text { mg }\end{array}$ & Leading AEs & $\begin{array}{r}\text { Incidence } \\
\text { of AEs, \% }\end{array}$ & $\begin{array}{l}\text { Withdrawal } \\
\text { due to AE, \% }\end{array}$ \\
\hline \multicolumn{5}{|c|}{ Randomized controlled study } \\
\hline Kawai et al. (2006) [6] & 3 & GI system, skin and appendages, body as a whole & 65 & 11.7 \\
\hline Kondo et al. (2004) [7] & 1.5 or 3 & Urogenital, GI system, liver and biliary & $44 \cdot 4$ & $4 \cdot 3$ \\
\hline Yocum et al. (2003) [8] & 2 or 3 & GI system, respiratory symptom, nervous & $>80$ & 13.4 \\
\hline \multirow{3}{*}{ Furst et al. (2002) [9] } & 1 & GI system, body as a whole, nervous & 92.8 & 10.1 \\
\hline & 3 & & 90.6 & 18.8 \\
\hline & 5 & & 89.1 & 12.5 \\
\hline \multicolumn{5}{|l|}{ Open-label study } \\
\hline Kawai et al. (2006) [12] & $1.5-3$ & Skin and appendages, GI system, body as a whole & 46.3 & 12.3 \\
\hline Yocum et al. (2004) [13] & 2 or 3 & GI system, nervous, respiratory & 59 & 16.2 \\
\hline Kremer et al. (2003) [14] & 3 & GI system, respiratory, nervous & 86.3 & 12.5 \\
\hline Present study & 1.5 & GI system, infection, musculoskeletal & 66.1 & $5 \cdot 4$ \\
\hline
\end{tabular}

AEs, adverse events; TAC, tacrolimus; GI, gastrointestinal.

tients who have comorbidities such as severe interstitial lung disease, nontuberculous mycobacterial infection, chronic hepatitis B infection, and cachexia. In addition, some patients refuse biologics owing to fear of injection or for financial reasons.

Reducing the disease activity of RA is the main strategy for obtaining a long-term favorable outcome. In this study, the addition of tacrolimus ( $1.5 \mathrm{mg}$ ) to MTX ther- apy was found to result in significant improvements in the relief and control of rheumatoid symptoms, as determined by the ACR response rate and health condition of RA subjects with active RA who showed an inadequate response to MTX. In such patients, $1.5 \mathrm{mg}$ of tacrolimus may be a good therapeutic option.

In conclusion, as RA is not a uniform disease, and since patients react differently to different drugs, a variety of 
treatment options for RA are needed. The present study showed that the addition of tacrolimus (1.5 mg) to MTX in RA patients who responded inadequately to MTX was an effective and safe treatment option. Accordingly, we believe that this combination therapy is a useful alternative RA treatment, and further large-scale, open-label as well as blinded, placebo-controlled studies should be performed to confirm our findings, both in Korean and international populations.

\section{KEY MESSAGE}

1. The addition of tacrolimus $(1.5 \mathrm{mg})$ to methotrexate (MTX) in rheumatoid arthritis patients who responded inadequately to MTX was an effective and safe treatment option.

\section{Conflict of interest}

No potential conflict of interest relevant to this article was reported.

\section{REFERENCES}

1. Moreland LW, O'Dell JR, Paulus HE, et al. A randomized comparative effectiveness study of oral triple therapy versus etanercept plus methotrexate in early aggressive rheumatoid arthritis: the treatment of Early Aggressive Rheumatoid Arthritis Trial. Arthritis Rheum 2012;64:28242835 .

2. Goekoop-Ruiterman YP, de Vries-Bouwstra JK, Allaart CF, et al. Clinical and radiographic outcomes of four different treatment strategies in patients with early rheumatoid arthritis (the BeSt study): a randomized, controlled trial. Arthritis Rheum 2005;52:3381-3390.

3. Ozminkowski RJ, Burton WN, Goetzel RZ, Maclean R, Wang $\mathrm{S}$. The impact of rheumatoid arthritis on medical expenditures, absenteeism, and short-term disability benefits. J Occup Environ Med 2006;48:135-148.

4. Kino T, Hatanaka H, Miyata S, et al. FK-506, a novel immunosuppressant isolated from a Streptomyces. II. Immunosuppressive effect of FK-506 in vitro. J Antibiot (Tokyo) 1987;40:1256-1265.

5. McDiarmid SV, Colonna JO 2nd, Shaked A, Vargas J, Ament ME, Busuttil RW. Differences in oral FK506 dose requirements between adult and pediatric liver trans- plant patients. Transplantation 1993;55:1328-1332.

6. Kawai S, Hashimoto H, Kondo H, Murayama T, Kiuchi T, Abe T. Comparison of tacrolimus and mizoribine in a randomized, double-blind controlled study in patients with rheumatoid arthritis. J Rheumatol 2006;33:2153-2161.

7. Kondo H, Abe T, Hashimoto H, et al. Efficacy and safety of tacrolimus (FK506) in treatment of rheumatoid arthritis: a randomized, double blind, placebo controlled dose-finding study. J Rheumatol 2004;31:243-251.

8. Yocum DE, Furst DE, Kaine JL, et al. Efficacy and safety of tacrolimus in patients with rheumatoid arthritis: a double-blind trial. Arthritis Rheum 2003;48:3328-3337.

9. Furst DE, Saag K, Fleischmann MR, et al. Efficacy of tacrolimus in rheumatoid arthritis patients who have been treated unsuccessfully with methotrexate: a six-month, double-blind, randomized, dose-ranging study. Arthritis Rheum 2002;46:2020-2028.

10. Arnett FC, Edworthy SM, Bloch DA, et al. The American Rheumatism Association 1987 revised criteria for the classification of rheumatoid arthritis. Arthritis Rheum 1988;31:315-324.

11. Felson DT, Anderson JJ, Boers M, et al. American College of Rheumatology. Preliminary definition of improvement in rheumatoid arthritis. Arthritis Rheum 1995;38:727-735.

12. Kawai S, Yamamoto K. Safety of tacrolimus, an immunosuppressive agent, in the treatment of rheumatoid arthritis in elderly patients. Rheumatology (Oxford) 2006;45:441-444.

13. Yocum DE, Furst DE, Bensen WG, et al. Safety of tacrolimus in patients with rheumatoid arthritis: long-term experience. Rheumatology (Oxford) 2004;43:992-999.

14. Kremer JM, Habros JS, Kolba KS, et al. Tacrolimus in rheumatoid arthritis patients receiving concomitant methotrexate: a six-month, open-label study. Arthritis Rheum 2003;48:2763-2768.

15. Gerards AH, Landewe RB, Prins AP, et al. Cyclosporin A monotherapy versus cyclosporin A and methotrexate combination therapy in patients with early rheumatoid arthritis: a double blind randomised placebo controlled trial. Ann Rheum Dis 2003;62:291-296.

16. Tugwell P, Bombardier C, Gent M, et al. Low-dose cyclosporin versus placebo in patients with rheumatoid arthritis. Lancet 1990;335:1051-1055.

17. Smolen JS, Landewe R, Breedveld FC, et al. EULAR recommendations for the management of rheumatoid arthritis with synthetic and biological disease-modifying 
antirheumatic drugs. Ann Rheum Dis 2010;69:964-975.

18. Visser K, Katchamart W, Loza E, et al. Multinational evidence-based recommendations for the use of methotrexate in rheumatic disorders with a focus on rheuma- toid arthritis: integrating systematic literature research and expert opinion of a broad international panel of rheumatologists in the $3 \mathrm{E}$ Initiative. Ann Rheum Dis 2009;68:1086-1093. 